Ciência Florestal, Santa Maria, v. 24, n. 3, p. 677-684, jul.-set., 2014

ISSN 0103-9954

\title{
EFEITO DA QUALIDADE DA MADEIRA NO DESEMPENHO DA POLPAÇÃO KRAFT
}

\author{
WOOD QUALITY EFFECT ON KRAFT PULPING PERFORMANCE
}

\author{
Danila Morais de Carvalho ${ }^{1}$ Marcelo Rodrigues da Silva² Jorge Luiz Colodette ${ }^{3}$
}

\begin{abstract}
RESUMO
O objetivo do estudo foi avaliar o efeito da qualidade de madeiras de eucalipto no desempenho da polpação kraft sob duas diferentes condições de polpação e em dois níveis de deslignificação, baseando-se no rendimento, alvura, viscosidade e índice $\mathrm{k} / \mathrm{kappa}$ corrigido das polpas produzidas. As madeiras de eucalipto (A, B e C), foram analisadas quanto às suas propriedades físico-químicas (densidades básica e aparente, teor de ligninas insolúvel e solúvel, teor de extrativos, cinzas e cinzas insolúveis em ácido clorídrico) e utilizadas para a produção de polpa celulósica por meio do cozimento kraft modificado, utilizando duas condições de polpação, sendo elas: Condição I (PI) - álcali efetivo de 16,5\%, sulfidez de $30 \%$ e temperatura de cozimento de $155^{\circ} \mathrm{C}$; e Condição II (PII) - álcali efetivo de $17,5 \%$, sulfidez de $32 \%$ e temperatura de cozimento de $147^{\circ} \mathrm{C}$. Ambos os cozimentos foram realizados buscando-se no número kappa de 15,5 $\pm 0,5 \mathrm{e}$ de 18,0 $\pm 0,5$. Foram avaliados os seguintes parâmetros de polpação e propriedades das polpas produzidas: fator $\mathrm{H}$, rendimento depurado, viscosidade, alvura, conteúdo de ácidos hexenurônicos e o índice $\mathrm{k} / \mathrm{kappa}$ corrigido. A madeira A, que apresentou menores teores de extrativos e lignina em sua constituição, bem como menores densidades básica e aparente quando comparada com as madeiras B e C, apresentou os melhores resultados para os parâmetros de cozimento avaliados, com destaque para a polpa produzida pela condição de cozimento PII com o número kappa de $(18,0 \pm 0,5)$, que apresentou maior rendimento, alvura e viscosidade dentre todas as polpas produzidas. Polpas produzidas com maior número kappa apresentam menores índices $\mathrm{k} /$ kappa corrigidos.
\end{abstract}

Palavras-chave: Eucalyptus; qualidade da madeira; grau de deslignificação; condições de polpação kraft.

\section{ABSTRACT}

The aim of this study was to evaluate the wood quality in the kraft pulping performance under two different pulping conditions at two delignification degrees based on results for the yield, brightness, viscosity, and k/ kappa corrected index. Eucalyptus wood (A, B, and C) was analyzed about their physicochemical properties (basic and apparent density, insoluble and soluble lignin content, extractive content, ash and insoluble ash in hydrochloric acid) and used for pulp production by modified kraft cooking, using two different pulping conditions: Condition I (PI) - 16.5\% effective alkali, 30\% sulfidity, and $155^{\circ}$ of cooking temperature; and Condition II (PII) - $17.5 \%$ effective alkali, $32 \%$ sulfidity, and $147^{\circ} \mathrm{C}$ cooking temperature. Both conditions were performed to kappa number $15.5 \pm 0.5$ and $18.0 \pm 0.5$. The following pulping parameters and pulp properties produced were evaluated: $\mathrm{H}$ factor, screened yield, viscosity, brightness, hexenuronic acid content, and the k/kappa corrected index. The A wood, which had lower extractives and lignin content in its constitution, as well as lower basic and apparent density when compared with B and C woods, showed good results for cooking parameters, especially the pulp produced at PII condition at kappa number $18.0 \pm$ 0.5.Also, the A wood showed higher screened yield, brightness, and viscosity comparing to the other pulps produced. Those pulps produced with higher kappa number have lower k/kappa corrected index.

Keywords: Ecalyptus; wood quality; delignification degree; kraft pulping conditions.

1 Engenheira Florestal, Msc., Doutoranda em Ciência Florestal, Departamento de Engenharia Florestal, Universidade Federal de Viçosa, Campus Universitário, CEP 36570-900, Viçosa (MG), Brasil. carvalho.danila@gmail.com

2. Engenheiro Químico, Dr., Especialista de Processo da Empresa Fibria Unidade Jacareí, Rod. Gal Euryale Jesus Zerbini, Km 84, SP 66, São Silvestre, CEP 12340-010, Jacareí(SP), Brasil. marcelo.silva@fibria.com.br

3 Engenheiro Florestal, PhD., Professor Titular do Departamento de Engenharia Florestal, Universidade Federal de Viçosa, Campus Universitário, CEP 36570-900, Viçosa (MG), Brasil. colodett@ufv.br

Recebido para publicação em 30/04/2011 e aceito em 26/03/2013

Ci. Fl., v. 24, n. 3, jul.-set., 2014 


\section{INTRODUÇÃO}

A grande diversidade de qualidade da matéria-prima e de tecnologias disponíveis para a produção de celulose branqueada de eucalipto requer estudos aprofundados e um melhor entendimento da interação entre elas para se tirar melhor proveito desse importante recurso fibroso.

$\mathrm{O}$ processo de produção de celulose branqueada é dividido em três etapas. A primeira delas é a escolha da madeira, na qual clones selecionados são cultivados, a fim de suprir a demanda de madeira da fábrica. Mesmo que haja um rigoroso processo de seleção do material genético, ainda assim é possível verificar diferenças quantitativas e qualitativas no que diz respeito aos componentes formadores da madeira. Para Latorraca e Albuquerque (2000), as propriedades da madeira são decorrentes das suas características genéticas e da influência que esta sofre do ambiente onde se desenvolve. Desta forma, é importante conhecer as características do material a ser trabalhado para melhor definir os tratamentos aos quais ele será submetido, bem como compreender os resultados obtidos.

A segunda etapa do processo é a polpação kraft, na qual o principal objetivo é separar as fibras de celulose por meio da remoção da lignina. Não apenas a quantidade de lignina interfere na dinâmica e eficiência da polpação, mas o tipo de lignina também influencia no grau de deslignificação e/ou economia do processo. Outra variável associada a polpação e a qualidade da madeira é a formação dos ácidos hexenurônicos a partir dos ácidos urônicos presentes em quantidade considerável na madeira de eucalipto. De acordo com Jiang et al. (2000), os ácidos urônicos encontram na polpação kraft ccondições ideais para reagir formando ácidos hexenurônicos, compostos que prejudicam o processo de branqueamento por consumir parte dos reagentes utilizados, além de comprometer a qualidade da polpa branqueada produzida se eles não forem retirados da polpa durante o branqueamento.

Por fim, a terceira etapa consiste em, através de sequências definidas, branquear a polpa produzida pela etapa de polpação. Diversos reagentes químicos podem ser utilizados durante a etapa de branqueamento, assim como diferentes tempos, $\mathrm{pH}$ e temperaturas, que são definidos para cada um destes reagentes, de modo a balancear a utilização mais eficiente de cada reagente com a menor perda de qualidade da polpa.
O objetivo desse estudo foi investigar o efeito da qualidade da madeira, das condições de cozimento e do grau de deslignificação da polpa no desempenho da polpação kraft de eucalipto, com foco no rendimento do processo, viscosidade, alvura e índice $\mathrm{k} / \mathrm{kappa}$ corrigido das polpas.

\section{MATERIAIS E MÉTODOS}

Este estudo foi realizado no Centro de Tecnologia e Pesquisa da Fibria S/A - Unidade Jacareí/SP. Foram utilizadas três amostras de madeiras: madeira A (híbrido de Eucalyptus urophylla e E. grandis + E. grandis, com 7 anos de idade, provenientes de plantios no estado de São Paulo), madeira B (mix de madeiras de híbrido de E. urophylla e E. grandis, com 10 anos de idade, provenientes de plantios no Mato Grosso do Sul) e madeira C (híbrido de E. urophylla e E. grandis, com aproximadamente 7 anos de idade, proveniente do estado da Bahia). Os cavacos das madeiras A e $\mathrm{C}$ foram coletados nas correias transportadoras que alimentam o digestor, tendo sido picados em picador industrial. A madeira $\mathrm{B}$ foi recebida em forma de toras e os cavacos foram produzidos em picador laboratorial. Estas amostras de madeira foram analisadas quanto às suas características químicas (extrativos totais, lignina solúvel, insolúvel e total, holocelulose, cinzas, insolúveis em $\mathrm{HCl}$ e metais) e físicas (densidades básica e aparente).

Os cozimentos foram realizados em triplicata, com o equivalente a 1400 gramas absolutamente secos (g. a. s.) de cavacos, com teor de umidade inferior a $15 \%$ e previamente classificados em peneiras vibratórias. A remoção de casca e nós dos cavacos foi feita manualmente.

Antes do processo de polpação foi feita a etapa de vaporização nos cavacos. A execução desta etapa consistiu na injeção de vapor a $100^{\circ} \mathrm{C}$ nos cavacos, já pesados, por um tempo de 15 minutos. Após a vaporização, os cavacos foram pesados e a quantidade de vapor de água retida nos cavacos foi descontada da quantidade de água a ser adicionada para suprir a relação licor/madeira dos cozimentos, que foi fixada em 3,5.

As polpas foram produzidas através da simulação de cozimentos Lo-Solids ${ }^{\mathrm{TM}}$, conforme procedimento do Centro de Tecnologia e Pesquisa (Fibria -Jacareí), efetuando-se, além da dosagem de alimentação, mais duas dosagens de licor branco durante o cozimento. Desta forma, o cozimento foi dividido em: fase de impregnação, fase de 
cozimento I e fase de cozimento II. Os cozimentos foram realizados em reatores MK, acoplados a vasos de armazenamento de licor.

Para cada amostra de cavacos, os cozimentos foram realizados buscando dois níveis de número kappa: $15,5 \pm 0,5$ e $18,0 \pm 0,5$. O valor de número kappa 15,5 $\pm 0,5$ era o utilizado pela Fibria - Jacareí no início do desenvolvimento deste estudo e o valor de 18,0 $\pm 0,5$ era o número kappa das polpas marrons das empresas de celulose no país. Para cada nível de número kappa foram utilizadas duas condições de cozimento, a Condição I (PI) e a Condição II (PII) (Tabela 1), onde, a partir da padronização das condições, o tempo de cozimento foi o único parâmetro influente no fator $\mathrm{H}$ que pôde ser alterado a fim de se obter os dois valores de número kappa desejados. Estas polpas foram denominadas de PI 15, PI 18, PII 15 e PII 18, segundo a condição de cozimentos utilizada e o número kappa da polpa marrom. As condições de cozimento foram obtidas a partir de informações fornecidas por duas empresas de destaque na produção de celulose branqueada nacional, e foram utilizadas como parâmetro de referência na realização deste estudo.

Os licores de cozimento foram preparados em laboratório a partir de hidróxido de sódio fornecido pelo processo fabril e sulfeto de sódio sólido, com padronização do licor preparado antes de cada novo cozimento.

Após os cozimentos, as polpas foram lavadas e depuradas em depurador laboratorial da marca Voith, dotado de placa com fendas de $0,2 \mathrm{~mm}$, onde as fibras que passaram pelas fendas foram recolhidas e posteriormente pesadas para determinação do rendimento depurado do processo. Os feixes de fibras e pedaços de cavacos malcozidos retidos nas fendas foram quantificados para a determinação do teor de rejeitos do cozimento.

O processo de cozimento foi avaliado quanto ao fator $\mathrm{H}$, rendimento depurado, teor de rejeitos e rendimento total. Nas polpas depuradas foram analisados: número kappa, viscosidade, teor de ácidos hexenurônicos, alvura e índice k/kappa corrigido.

$\mathrm{O}$ índice $\mathrm{k} / \mathrm{kappa}$ corrigido foi obtido a partir da razão entre o valor de $\mathrm{k}$, índice de absorção de luz (um componente da alvura), pelo valor do número kappa corrigido, de modo que a contribuição do número kappa que é devido aos ácidos hexenurônicos foi desconsiderada (ALDAJANI, 2001). Neste estudo utilizaram-se os valores médios dos ácidos hexenurônicos de cada madeira e condição de cozimento para o cálculo do índice $\mathrm{k} / \mathrm{kappa}$ corrigido. $\mathrm{O}$ índice $\mathrm{k}$ é um componente da fórmula da alvura proposta por Kubelka-Munk (KUBELKA; MUNK, 1931 citado por GUSTAVSSON et al., 1999):

$$
\mathrm{R}_{\infty}=1+(\mathrm{k} / \mathrm{s})-(\mathrm{k} / \mathrm{s})^{2}+2(\mathrm{k} / \mathrm{s})
$$

Analisando os componentes da fórmula tem-se que: $\mathrm{R}_{\infty}$ representa a alvura, $\mathrm{k}$ é o índice de absorção de luz e s é o índice de espalhamento de luz. A radiação utilizada $(457 \mathrm{~nm})$ abrange apenas o comprimento de onda do visível, que é o comprimento de onda no qual a lignina tem a capacidade de absorver máxima radiação e, deste modo, este componente interfere no resultado. Para que o resultado do índice $\mathrm{k} / \mathrm{kappa}$ correlacione apenas com o conteúdo de lignina (residual e complexo lignina-carboidrato) da polpa, torna-se necessário corrigir este índice, desconsiderando-se a contribuição dos demais componentes quantificados no número kappa, sendo os principais componentes influentes no número kappa os ácidos hexenurônicos (COSTA; COLODETTE, 2001). Como as polpas foram misturadas para a confecção das folhinhas para a determinação do componente $\mathrm{k}$ deste índice, um novo número kappa foi determinado a partir da mistura destas polpas para ser utilizado na fórmula proposta.

Na Tabela 5 são apresentados os resultados

TABELA 1: Parâmetros dos cozimentos.

TABLE 1: Cooking parameters.

\begin{tabular}{lcc}
\hline \multicolumn{1}{c}{ Parâmetros dos cozimentos } & Condição PI & Condição PII \\
\hline Carga alcalina, AE \% como NaOH & $16,5(7,6 ; 1,8 ; 7,1)^{1}$ & $17,5(7,9 ; 4,9 ; 4,7)^{1}$ \\
Sulfidez, \% & 30 & 32 \\
Temperatura de cozimento, ${ }^{\circ} \mathrm{C}$ & 155 & 147 \\
Fator H & Variável & Variável \\
\hline
\end{tabular}

Em que: ${ }^{~}$ Distribuição da carga alcalina na alimentação, transferência e extração, respectivamente. 
das análises de densidade dos cavacos das madeiras A, B e C.

Segundo Li e Gellerstedt (1997) citados por Gustavsson et al. (2000), a contribuição dos ácidos hexenurônicos para o número kappa é de uma unidade de número kappa para cada 11,6 $\mu \mathrm{mol}$ de ácidos hexenurônicos presentes na polpa. Ao se descontar a contribuição dos ácidos hexenurônicos no número kappa, e após se obter a razão entre o $\mathrm{k}$ (índice de absorção de luz) e o número kappa corrigido, tem-se o valor estimado do índice $\mathrm{k} /$ kappa corrigido, de modo que, para uma mesma madeira, valores menores para este índice são mais desejáveis, uma vez que indicam um menor consumo de reagentes químicos de branqueamento e, com isso, melhores branqueabilidades (ALDAJANI, 2001).

Os procedimentos analíticos utilizados neste estudo estão citados na Tabela 2.

\section{RESULTADOS E DISCUSSÃO}

\section{Caracterização físico-química das madeiras}

A Tabela 3 apresenta os valores obtidos para a composição química orgânica das madeiras. Segundo Latorraca e Albuquerque (2000), estes constituintes são produtos da fotoassimiliação e sua síntese depende das condições climáticas do local onde a planta está se desenvolvendo.

Embora os valores obtidos não indiquem grandes diferenças de composição química entre madeiras, verifica-se que a madeira $\mathrm{A}$ apresentou os maiores valores de holocelulose e lignina solúvel e os menores valores de extrativos e lignina insolúvel. Este fato pode ser explicado devido à característica ainda jovem desta madeira (7 anos), na qual a lignina ainda se encontra menos condensada e mais facilmente removível. A madeira B apresentou os maiores valores de lignina insolúvel e de lignina total, muito provavelmente pelo fato de ter uma idade maior que das demais madeiras analisadas (10 anos), o que refletiu também nos menores teores de holocelulose.

A Tabela 4 mostra a composição química inorgânica das madeiras. Notou-se que os maiores percentuais de cinzas e minerais insolúveis em $\mathrm{HCl}$ são encontrados na madeira $\mathrm{A}$. Esta mesma madeira apresentou ainda maiores teores de cálcio, magnésio, ferro, alumínio e potássio. $\mathrm{O}$ sódio e manganês destacaram-se com seus altos e baixos teores, respectivamente, para a madeira C. Os teores de manganês e silício foram ligeiramente superiores para a madeira $\mathrm{B}$.

TABELA 2: Procedimentos analíticos para caracterização físico-química das madeiras e das polpas celulósicas.

TABLE 2: Analytical procedures for physical and chemical characterization of wood and pulps.

\begin{tabular}{ll}
\hline \multicolumn{1}{c}{ Parâmetros } & \multicolumn{1}{c}{ Procedimentos } \\
\hline Teor de extrativos totais & TAPPI T 264 cm-07 \\
Teor de lignina insolúvel em ácido & Goldschimid (1971) \\
Teor de lignina solúvel em ácido & Gomide e Demuner (1986) \\
Holocelulose & Calculada por diferença \\
Teor de cinzas & TAPPI 211 om 02 \\
Teor de insolúveis em HCl & TAPPI 244 cm-11 \\
Densidade básica & TAPPI T-258 om-06 \\
Densidade aparente & SCAN CM-46:92 \\
Classificação de cavacos & SCAN-CM 40:94 \\
Número kappa & TAPPI T 236 om-06 \\
Teor de ácidos hexenurônicos & Método interno Fibria -Jacareí \\
Alvura ${ }^{1}$ & TAPPI T 452 om-08 \\
Viscosidade & TAPPI T 230 om-08 \\
Índice k/kappa corrigido & 2 \\
\hline Em que: ${ }^{1}$ Foram realizados no aparelho DATACOLOR modelo ELREPHO 2000. ${ }^{2}$ Adaptado de Li e Gellerstedt (1997) \\
citado por Gustavsson et al. (1999).
\end{tabular}


TABELA 3: Composição química orgânica das madeiras.

TABLE 3: Wood organic chemical composition.

\begin{tabular}{cccc}
\hline Constituintes Orgânicos & Madeira A & Madeira B & Madeira C \\
\hline Teor de extrativos (\%) & 2,1 & 3,3 & 3,4 \\
Teor de lignina solúvel $^{1}(\%)$ & 4,1 & 3,3 & 3,6 \\
Teor de lignina insolúvel $^{1}(\%)$ & 24,0 & 26,2 & 24,9 \\
Teor de lignina total $^{1}(\%)$ & 28,1 & 29,4 & 28,5 \\
Teor de holocelulose $^{1}(\%)$ & 69,5 & 67,1 & 67,9 \\
\hline
\end{tabular}

Em que: ${ }^{1}$ Valores percentuais obtidos com base na madeira com extrativos.

TABELA 4: Composição química inorgânica das madeiras.

TABLE 4: Wood inorganic chemical composition.

\begin{tabular}{cccc}
\hline Constituintes Inorgânicos & Madeira A & Madeira B & Madeira C \\
\hline Teor de cinza (\%) & 0,35 & 0,21 & 0,20 \\
Teor de insolúveis em HCl (\%) & 0,039 & 0,013 & 0,013 \\
Ca (ppm) & 532 & 365 & 421 \\
Mg (ppm) & 103 & 90,2 & 95,1 \\
Fe (ppm) & 15,2 & 0,48 & 0,17 \\
Mn (ppm) & 38,4 & 44,9 & 5,3 \\
Al (ppm) & 12,4 & 4,2 & 4,5 \\
Si (ppm) & 10,0 & 11,6 & 8,6 \\
Na (ppm) & 51,4 & 48,0 & 155 \\
K (ppm) & 1217 & 767 & 777 \\
\hline
\end{tabular}

TABELA 5: Densidade básica e aparente dos cavacos.

TABLE5: Chip basic and apparent density.

\begin{tabular}{cccc}
\hline Densidades & Madeira A & Madeira B & Madeira C \\
\hline Básica $\left(\mathrm{kg} / \mathrm{m}^{3}\right)$ & 504 & 522 & 542 \\
Aparente $\left(\mathrm{kg} / \mathrm{m}^{3}\right)$ & 168 & 202 & 178 \\
\hline
\end{tabular}

Na Tabela 5 são apresentados os resultados das análises de densidade dos cavacos das madeiras A, B e C. As madeiras apresentaram diferentes densidades básicas e aparentes.

\section{Operações de cozimentos para obtenção de polpas marrons}

Foram produzidas polpas kraft de número kappa 15,5 $\pm 0,5$ e 18,0 $\pm 0,5$ sob as condições de cozimento PI e PII, apresentadas na Tabela 1. A condição de cozimento PII 15 não foi realizada para a madeira B. Os resultados médios para cada condição de polpação e número kappa estão apresentados nas Tabelas 6, 7 e 8, respectivamente, para as madeiras A, B e C.

Os cozimentos PII necessitaram os menores valores de fator $\mathrm{H}$ para alcançarem o número kappa desejado para a madeira A (Tabela 6), favorecendo assim os parâmetros de alvura e viscosidade. A tendência verificada para a viscosidade está de acordo com o reportado por Gomide et al. (2000), que afirmam que menores temperaturas de cozimento favorecem a preservação dos carboidratos da madeira durante a polpação.

A interrupção do cozimento em maior valor de número kappa forneceu melhores resultados de rendimento depurado e viscosidade. Os teores de 
TABELA 6: Resultados médios de polpação da madeira A.

TABLE 6: Average pulping results for A wood.

\begin{tabular}{ccccc}
\hline \multirow{2}{*}{ Resultados } & \multicolumn{4}{c}{ Condições de polpação - Madeira A } \\
\cline { 2 - 5 } & PI 15 & PI 18 & PII 15 & PII 18 \\
\hline Fator H & 624 & 525 & 520 & 335 \\
Rendimento depurado, \% & 53,7 & 54,8 & 53,7 & 54,9 \\
Teor de rejeito, \% & 0,01 & 0,05 & 0,01 & 0,03 \\
Rendimento total, \% & 53,7 & 54,8 & 53,7 & 54,9 \\
Viscosidade, $\mathrm{cm}^{3} / \mathrm{g}$ & 1377 & 1402 & 1400 & 1422 \\
Alvura, \% ISO & 42,6 & 41,2 & 45,2 & 43,4 \\
Teor de ácidos hexenurônicos, $\mu \mathrm{mol} / \mathrm{g}$ & 54,1 & 59,4 & 66,1 & 53,7 \\
Índice $\mathrm{k} / \mathrm{kappa}$ corrigido, $\mathrm{m}^{2} / \mathrm{kg}$ & 0,49 & 0,43 & 0,49 & 0,43 \\
\hline
\end{tabular}

ácidos hexenurônicos não apresentaram uma onde tendência clara quanto às condições de polpação e o grau de deslignificação, já que diversas variáveis interferem na formação e degradação destes ácidos, tempo de cozimento e carga alcalina atuam de forma conjunta neste processo. Segundo Daniel et al. (2003), nas etapas iniciais do cozimento, os ácidos hexenurônicos começam a ser formados e são mais rapidamente formados quanto maior for a temperatura e a carga alcalina. Com o desenvolvimento do processo de cozimento, o aumento do tempo e da temperatura favorece a degradação dos ácidos hexenurônicos gerados. Além disso, quanto maior a carga alcalina $\left[\mathrm{OH}^{-}\right]$, maior será a degradação dos ácidos hexenurônicos formados, como afirmam Gustavsson et al. (1999). A concentração de sulfeto de sódio não influencia diretamente na cinética da formação/degradação desses ácidos, no entanto, sua presença facilita a acessibilidade à lignina pelo licor de cozimento o que, por beneficiar a deslignificação, favorece também a reação dos ácidos hexenurônicos com o licor de cozimento, no sentido de sua degradação, uma vez que o sulfeto de sódio, por ser altamente seletivo, tende a proteger os carboidratos (SIXTA; RUTKOWSKA, 2007). Cada madeira e condição de cozimento apresentam tempos e temperaturas diferentes para que esta degradação comece a ocorrer. ocorrer. Tais argumentos descritos acima ajudaram para justificar o fato de que os valores de ácidos hexenurônicos não seguiram uma tendência clara, uma vez que, neste estudo, foram alteradas mais de uma variável relacionada à sua formação/ degradação, sendo elas: tipo de madeira, condição de cozimento e grau de deslignificação da polpa.
Os valores apresentados pelo índice $\mathrm{k} /$ kappa corrigido nas Tabelas 6,7 e 8 foram obtidos a partir da razão entre o $\mathrm{k}$ (índice de absorção de luz, que é um componente da alvura) e o número kappa corrigido a partir da exclusão da contribuição dos ácidos hexenurônicos no número kappa. Quanto menor o valor apresentado por este índice, maior é a branqueabilidade esperada para a polpa, ou seja, menor o consumo de reagentes químicos para se obter uma dada alvura final (AL-DAJANI, 2001). Deste modo, os melhores resultados para este parâmetro foram obtidos para as polpas produzidas com maior número kappa $(18,0 \pm$ $0,5)$, independentemente do tipo de madeira e condição de polpação, já que seus menores índices implicam em melhor capacidade de branqueamento com menor consumo de reagentes químicos. Este comportamento dos resultados de apresentarem uma correlação positiva entre número kappa e índice $\mathrm{k} /$ kappa corrigido está de acordo com o encontrado por Al-Dajani (2001) em seu estudo, onde esta mesma tendência foi verificada.

$\mathrm{Na}$ Tabela 7 encontram-se os resultados dos parâmetros de cozimentos relacionados às polpas produzidas a partir da madeira B. Os resultados encontrados para esta madeira diferiram da tendência apresentada para a madeira A (Tabela 6) e pela madeira $C$ (Tabela 8), mostrando que a condição de cozimento PII não apresenta para a madeira B a mesma resposta que apresentou para as demais madeiras. A dificuldade de polpação desta madeira pode ver verificada pelo elevado valor de fator $\mathrm{H}$ necessário para se alcançar os valores de número kappa desejados. Foi possível perceber que, quanto maior o valor do fator $\mathrm{H}$, maiores 
foram os valores encontrados para o índice $\mathrm{k} / \mathrm{kappa}$ corrigido, o que está de acordo com o reportado por Al-Dajani (2001), quando afirma que esse índice aumenta com o aumento do fator $\mathrm{H}$ do cozimento, tendência também verificada neste presente estudo. Os baixos rendimentos e maior fator $\mathrm{H}$ demandados por esta madeira, associados ao fato de não ter sido possível realizar a condição PII 15 indicaram que, não só a madeira apresentou resistência ao processo de cozimento, como também demandou maiores temperaturas para alcançar a separação das fibras, e que esta medida comprometeu a qualidade da polpa formada (menores viscosidade e alvura). Deste modo, a condição PII de polpação não foi suficiente para deslignificar a madeira, produzindo resultados menos animadores de rendimento depurado, viscosidade, alvura e índice $\mathrm{k} / \mathrm{kappa}$ corrigido para quando comparada à condição PI. Embora o valor de ácido hexenurônico apresentado pela condição
PII 18 tenha sido maior que o apresentado para as demais condições, não foi possível inferir uma tendência para estes valores por não se elucidar completamente a dinâmica dos ácidos hexenurônicos durante o cozimento com os dados obtidos neste estudo.

Analisando-se a Tabela 8 foi possível verificar que a condição PII demandou menores valores de fator $\mathrm{H}$ para a produção das polpas no número kappa de interesse. Notou-se que esta condição apresentou os melhores resultados de rendimento depurado, viscosidade, alvura e índice k/kappa corrigido, sendo esses parâmetros nitidamente influenciados pelo maior número kappa desejado. $\mathrm{O}$ fator $\mathrm{H}$ exerceu efeito positivo nos valores do índice k/kappa corrigido, o que está de acordo com o reportado por Al-Dajani (2001). Com exceção da alvura, os melhores resultados ocorreram quando se prodiu polpas com maior número kappa

TABELA 7: Resultados médios de polpação da madeira B.

TABLE 7: Average pulping results for B wood.

\begin{tabular}{ccccc}
\hline \multirow{2}{*}{ Resultados } & \multicolumn{4}{c}{ Condições de polpação - Madeira B } \\
\cline { 2 - 5 } & PI 15 & PI 18 & PII 15 & PII 18 \\
\hline Fator H & 817 & 571 & - & 614 \\
Rendimento depurado, \% & 51,5 & 52,0 & - & 50,7 \\
Teor de rejeito, \% & 0,00 & 0,01 & - & 0,00 \\
Rendimento total, \% & 51,5 & 52,0 & - & 50,7 \\
Viscosidade, $\mathrm{cm}^{3} / \mathrm{g}$ & 1295 & 1390 & - & 1385 \\
Alvura, $\%$ ISO & 38,1 & 36,9 & - & 39,3 \\
Teor de ácidos hexenurônicos, $\mu \mathrm{mol} / \mathrm{g}$ & 56,6 & 52,5 & - & 65,5 \\
Índice $\mathrm{k} /$ kappa corrigido, $\mathrm{m}^{2} / \mathrm{kg}$ & 0,77 & 0,57 & - & 0,61 \\
\hline
\end{tabular}

TABELA 8: Resultados médios de polpação da madeira C.

TABLE 8: Average pulping results for $\mathrm{C}$ wood.

\begin{tabular}{ccccc}
\hline \multirow{2}{*}{ Resultados } & \multicolumn{4}{c}{ Condições de polpação - Madeira C } \\
\cline { 2 - 5 } & PI 15 & PI 18 & PII 15 & PII 18 \\
\hline Fator H & 753 & 525 & 572 & 396 \\
Rendimento depurado, \% & 52,7 & 54,1 & 53,4 & 54,1 \\
Teor de rejeito, \% & 0,01 & 0,04 & 0,00 & 0,06 \\
Rendimento total, \% & 52,7 & 54,0 & 53,4 & 54,2 \\
Viscosidade, $\mathrm{cm}^{3} / \mathrm{g}$ & 1259 & 1377 & 1349 & 1386 \\
Alvura, \% ISO & 42,3 & 40,8 & 42,7 & 41,0 \\
Teor de ácidos hexenurônicos, $\mu \mathrm{mol} / \mathrm{g}$ & 61,0 & 63,7 & 56,6 & 60,4 \\
Índice $\mathrm{k} / \mathrm{kappa} \mathrm{corrigido,} \mathrm{m}^{2} / \mathrm{kg}$ & 0,60 & 0,49 & 0,50 & 0,47 \\
\hline
\end{tabular}


$(18,0 \pm 0,5)$. Os melhores resultados de rendimento depurado apresentados pela condição de cozimento PII podem ser explicados pela menor temperatura de cozimento requerida para esta condição, o que está de acordo com o reportado em trabalhos de Ventorim et al. (2009) e Gomide et al. (2000). Os teores de ácidos hexenurônicos não apresentaram tendência clara em relação ao grau de deslignificação e condição de cozimento PI ou PII.

Dentre as madeiras, os melhores resultados, quanto aos diversos parâmetros (fator $\mathrm{H}$, rendimento depurado, viscosidade, alvura e índice k/kappa corrigido), foram verificados para a madeira $\mathrm{A}$. A madeira B apresentou os resultados menos animadores quanto a estes parâmetros (composição química e cozimentos). A madeira $\mathrm{C}$ apresentou resultados intermediários.

\section{CONCLUSÕES}

As madeiras apresentaram composições químicas distintas. Esses resultados podem ser explicados pelas diferenças verificadas na procedência, no material genético e na idade destas madeiras.

A madeira A apresentou os melhores resultados para todos os parâmetros avaliados.

Os menores valores de índice $\mathrm{k} / \mathrm{kappa}$ corrigido foram obtidos para as polpas de número kappa 18,0 $\pm 0,5$, independentemente da madeira e da condição de cozimento utilizada. É de se esperar que estas polpas sejam mais facilmente branqueáveis que as polpas produzidas com número kappa da polpa marrom de 15,5 $\pm 0,5$, que apresentam maiores valores para esse índice.

As várias madeiras apresentaram respostas distintas para as diferentes condições de polpação e graus de deslignificação, sugerindo a necessidade de avaliações específicas para cada madeira.

\section{AGRADECIMENTOS}

Os autores agradecem à Fibria S/A Unidade Jacareí, pelo espaço físico e suporte técnico fornecidos durante o desenvolvimento desse estudo.

\section{REFERÊNCIAS BIBLIOGRÁFICAS}

AL-DAJANI, W. W. On the bleachability of alkaline pulps.The influence of residual lignin structure. 78 f. Doctoral Dissertation - Royal Institute of Technology (KTH) Departament of Pulp and Paper Chemistry and Technology, Stockholm, 2001.

COSTA, M. M.; COLODETTE, J. L. Efeito da composição química da polpa kraft $-\mathrm{O}_{2}$ na sua branqueabilidade. In: CONGRESSO ANUAL DE CELULOSE E PAPEL, 34., 2001, São Paulo. Anais... São Paulo: ABTCP, 2001. Não paginado.

DANIEL, A. I. D.; et al. Hexenuronic acid contents of Eucalyptus globuluskraft pulps: variation with pulping condition and effect on ECF bleachability. Tappi Journal, v. 2, n. 5, p. 3-8, 2003.

GOLDSCHIMID, O. Ultravioleta Spectra. In: SARKANEM, K.; LUDWING, C. H. Lignins: ocurrence, formation, structure and reactions. New York: John Wiley\& Sons, 1971. p. 241-298.

GOMIDE, J. L.; DEMUNER, B. J. Determinação do teor de lignina na madeira: método Klason modificado. O Papel, São Paulo, v. 47, n. 8, p. 36- 38, 1986.

GOMIDE, J. L. et al. Fatores que afetam a branqueabilidade de polpas Kraft de Eucalyptus 2: Influência de parâmetros da polpação. In: CONGRESSO INTERNACIONAL DE CELULOSE E PAPEL, 2000, São Paulo. Anais... São Paulo: ABTCP-TAPPI, 2000. Não paginado.

GUSTAVSSON, C. et al, W.W.The influence of cooking conditions on the bleachability and chemical structure of kraft pulps.Nordic Pulp and Paper Research Journal, v. 14, n. 1, p. 71-81, 1999. JIANG, Z. et al. Hexenuronic acid groups in pulping and bleaching chemistry. Tappi Journal, v. 83, n. 1, p. $167-175,2000$.

LATORRACA, J. V. F.; ALBUQUERQUE, C. E. C. Efeito do rápido crescimento sobre as propriedades da madeira. Floresta e Ambiente, v. 7, n. 1, p. 279-291, 2000.

SCAN-test Standard Scandinavian Pulp, Paper and Board Testing Committee, Stockholm, 1994.

SIXTA, H.; RUTKOWSKA, E. W. Estudo cinético abrangente da polpa kraft de Eucalyptusglobulus. O

Papel, São Paulo, v. 48, n. 2, p. 68-81, 2007.

TTECHINICAL ASSOCIATION OF THE PULP AND PAPER INDUSTRY - TAPPI. Tappi Standard Methods, Atlanta: TAPPI, 2011. VENTORIM, G. et al. A influência dos ácidos hexenurônicos no rendimento e na branqueabilidade da polpa kraft. Química Nova, v. 32, n. 2, p. 373-377, 2009. 